\title{
CONE BUNDLES
}

\author{
BY \\ CLINT MCCRORY $\left({ }^{1}\right)$
}

\begin{abstract}
A theory of normal bundles for locally knotted codimension two embeddings of PL manifolds is developed. The classifying space for this theory is Cappell and Shaneson's space $B R N_{2}$.
\end{abstract}

Cone bundles are a generalization of blockbundles [9], allowing local knotting of the base in the total space. They are a type of "mockbundle" [8], [2], closely related to the theory of stratified polyhedra [12], and designed to provide a simple foundation for Cappell and Shaneson's theory of singularities of PL embeddings [3], [4]. A similar definition has been given by Matumoto and Matsumoto [6].

$\$ 1$ contains the basic definitions. A classifying space for cone bundles is constructed in $\$ 2$. $\$ 3$ contains a proof that the total space of a cone bundle over a manifold is a manifold. Finally, cone bundles are related to the topology of stratified polyhedra in $\S 4$.

The geometric idea for cone bundles comes from my paper [7] on cone complexes. I thank Sylvain Cappell for encouraging me to develop this idea.

I will work in the category of polyhedra and piecewise linear maps [11]. In particular, all manifolds and homeomorphisms will be piecewise linear.

1. Thickenings. Let $M$ be a compact $n$-manifold. A codimension $q$ thickening of $M$ is a compact $(n+q)$-manifold $W$ containing $M$ as a subpolyhedron, such that $W$ collapses to $M$. Furthermore, $\partial W \cap M=\partial M$, and there is a collar of $\partial W$ in $W$ which restricts to a collar of $\partial M$ in $M$. (This is called a "very proper" thickening in [4].)

The thickenings $V$ and $W$ of $M$ are equivalent if there is a homeomorphism between $V$ and $W$ which is the identity on $M$.

If $q>2$, a codimension $q$ thickening $W$ of $M$ is an "abstract regular neighborhood" of $M$ [9, p. 14], since $M$ is locally flat in $W$. But if $q=2, M$ can be locally knotted in $W . M$ can be locally knotted in a codimension one thickening if and only if the PL Schoenflies conjecture is false.

Received by the editors November 20, 1975.

AMS (MOS) subject classifications (1970). Primary 57C40, 57C50; Secondary 57C45.

Key words and phrases. Mockbundle, blockbundle, locally knotted embedding, thickening, cone complex, piecewise linear stratification.

(') Supported in part by NSF Grant GP-43128.

(1) American Mathematical Society 1977 
Let $K$ be a (PL) cell complex. A $q$-cone bundle $\xi / K$ consists of a polyhedron $E(\xi)$ containing $|K|$ such that

(i) For each $p$-cell $\sigma_{i} \in K$ there is a $(p+q)$-ball $\beta_{i} \subset E(\xi)$, containing $\sigma_{i}$, such that $\left(\beta_{i}, \sigma_{i}\right)$ is homeomorphic with the cone on a sphere pair. (N.B. this sphere pair may be nonlocally flat or knotted.) $\beta_{i}$ is called the block over $\sigma_{i}$.

(ii) $E(\xi)$ is the union of the blocks $\beta_{i}$.

(iii) The interiors of the blocks are disjoint.

(iv) $\beta_{i} \cap \beta_{j}$ is the union of the blocks over the cells contained in $\sigma_{i} \cap \sigma_{j}$.

By the Zeeman unknotting theorem, a $q$-cone bundle is a blockbundle [9] if $q>2$, i.e., $\left(\beta_{i}, \sigma_{i}\right)$ is homeomorphic with a standard sphere pair for each $\sigma_{i} \in K$. The following results show that cone bundles bear essentially the same relation to thickenings that blockbundles bear to abstract regular neighborhoods.

LEMMA 1. Let $N$ be a manifold containing the manifold $M$ as a subpolyhedron. Suppose that $\partial N \cap M=\partial M$ and $(\partial N, \partial M)$ is collared in $(N, M)$. Then there is a cone bundle $\xi / K$ with $|K|=M$ such that $E(\xi)$ is a regular neighborhood of $M$ in $N$.

PROof. $\xi$ is constructed in the same manner as a normal blockbundle for a locally flat submanifold, using dual cells. (The usual construction must be slightly modified near the boundary; cf. [7, p. 284].)

LEMMA 2. If $\xi / K$ is a cone bundle, and $|K|$ is a compact manifold, then $E(\xi)$ is a thickening of $K$.

Proof. It is easy to see that $E(\xi)$ collapses to $|K|$, since $E(\xi)$ can be triangulated as a stellar neighborhood of $|K|$, by induction on the dimension of $\xi$ (cf. [7, p. 274]). The fact that $E(\xi)$ is a manifold will be proved in $\$ 3$.

LEMma 3. If $W$ is a thickening of the compact manifold $M$, there is a cone bundle $\xi / K,|K|=M$, such that the thickening $E(\xi)$ of $M$ is equivalent to $W$.

PRoof. This follows from Lemma 1 and the uniqueness of regular neighborhoods [11, p. 33].

REMARKs. (1) If $\xi / K$ and $\eta / L$ are cone bundles with $|K|=|L|=M$, and the thickenings $E(\xi)$ and $E(\eta)$ of $M$ are equivalent, one might expect (by analogy with blockbundles) that $\xi$ and $\eta$ have isomorphic "subdivisions". However, this is not true-one has to introduce the weaker relation of concordance (\$2) in order to get a bijection between classes of bundles and classes of thickenings.

(2) In Matumoto and Matsumoto's definition of “ $R N_{2}$-bundles" [6], condition (i) in the definition of a 2-cone bundle is weakened to the condition that $\left(\beta_{i}, \sigma_{i}\right)$ is an arbitrary ball pair. Lemma 2 is not true for their bundles, since the total space need not collapse to the base space. 
(3) A 2-cone bundle $\xi / K$ has a canonical "Noguchi characteristic class" $\mathfrak{n} \in H^{2}(K ; \gamma)$ (twisted coefficients), where $\gamma$ is the Fox-Milnor cobordism group (cf. [4]). $\mathfrak{n}$ is represented by the cocycle which assigns to each 2-cell $\sigma \in K$, the cobordism class of the knot $(\partial \beta, \partial \sigma)$, where $\beta$ is the block over $\sigma$. Thus $\mathfrak{n}$ is the primary obstruction to making $\xi$ a blockbundle. (The analogous higher obstructions are not defined a priori since $\partial \sigma_{i}$ is not necessarily locally flat in $\partial \beta_{i}$ if $\operatorname{dim} \sigma_{i}>2$.)

2. A classifying space. The following definitions come from [9].

If $\xi / K$ is a cone bundle and $L$ is a subcomplex of $K$, the restriction $\xi \mid L$ is defined by putting $\beta_{i}(\xi \mid L)=\beta_{i}(\xi)$ for each $\sigma_{i} \in L$.

The cone bundles $\xi_{0}, \xi_{1} / K$ are isomorphic if there is a homeomorphism $h$ : $E\left(\xi_{0}\right) \rightarrow E\left(\xi_{1}\right)$ such that $h$ is the identity on $|K|$ and $h\left(\beta_{i}\left(\xi_{0}\right)\right)=\beta_{i}\left(\xi_{1}\right)$ for each $\sigma_{i} \in K$.

The cone bundles $\xi_{0}, \xi_{1} / K$ are concordant if there is a cone bundle $\eta /(K \times I)$ such that $\eta \mid(K \times\{i\})$ is isomorphic with $\xi_{i}, i=0$, l. Here $I=[0$, 1] and $K \times I$ is the usual product complex. (Two blockbundles are concordant if and only if they are isomorphic $[9, \mathrm{p} .6]$. This is not true for 2-cone bundles.)

We will construct a classifying space for concordance classes of 2-cone bundles analogous to the classifying space $B \widetilde{P L}_{q}$ for $q$-blockbundles. (The same construction also works for 1-cone bundles.)

Let $\mathcal{H}(K)$ be the set of concordance classes of 2 -cone bundles over $K$. $\mathcal{H}$ is a contravariant functor from the category with objects PL cell complexes and morphisms generated by isomorphisms and inclusions of subcomplexes, to the category of (based) sets. (The base point of $\mathcal{H}(K)$ is the class of the trivial bundle over $K$.)

THEOREM 1. $\mathcal{H}$ has a unique extension to the category of $C W$ complexes and homotopy classes of maps.

Proof. This is a corollary of the "mockbundle" recipe for homotopy functors [2, I]. It is clear that cone bundles can be glued (axiom G [2, p. 15]), so we only have to verify the extension axiom (E, [2, p. 15]). That is, if $e$ : $K_{0} \rightarrow K$ is an elementary expansion, and $\xi_{0} / K_{0}$ is a cone bundle, we must construct a cone bundle $\xi / K$ such that $\xi \mid K_{0}=\xi_{0}$. We follow [2, p. 21]. $K=K_{0} \cup\{\sigma, \tau\}$, where $\sigma$ is a principal cell of $K$ and $\tau$ is a free face of $\sigma$. Let $J$ be the subcomplex of $\partial \sigma$ consisting of all the faces of $\sigma$ except $\tau$. Now $|J|$ is a ball, and $E\left(\xi_{0} \mid J\right)$ is a thickening of $|J|$ by Lemma 2, so $E\left(\xi_{0} \mid J\right)$ is a ball. Let $(B, C)$ denote the ball pair $\left(E\left(\xi_{0} \mid J\right),|J|\right)$. Identifying $(\sigma, C, \tau)$ with $(C \times I$, $C \times\{0\},(\partial C \times I) \cup(C \times\{1\}))$, we define the extension $\xi / K$ as follows. The block of $\xi$ over $\sigma$ is the cone on the boundary of the ball $B \times I$, and the block over $\tau$ is the cone on the boundary of the ball 


$$
\operatorname{cl}\left[\partial(B \times I) \backslash\left(B \cup\left(B^{*} \times I\right)\right)\right],
$$

where $B^{*}=\operatorname{cl}\left[\partial B \backslash \cup \beta_{i}\left(\xi_{0}\right)\right]$, union over all $i$ such that $\sigma_{i} \subset \partial C$.

THEOREM 2. $\mathcal{H}$ is a representable functor.

Proof. Let $G$ be the (based) $\Delta$-set whose $k$-simplexes are 2-cone bundles over $\Delta^{k}$ (the standard $k$-simplex) which are embedded blockwise in $\Delta^{k} \times R^{\infty}$, and let $\gamma$ be the canonical cone bundle on $G$ (cf. [8, p. 131] and [2, p. 37]). $G$ is a Kan $\Delta$-set by the extension axiom (see the proof of Theorem 1) and general position. It follows that if $\mathcal{G}$ is the realization of $G$, pulling back the class of $\gamma$ induces a bijection

$$
\mathcal{H}(X) \cong[X, \mathcal{G}]
$$

for all $C W$ complexes $X$, where [, ] denotes homotopy classes of maps (cf. $[10, \S 6]$ and $[9, \S 2])$.

Theorems 1 and 2 are also true for Matumoto and Matsumoto's $R N_{2}$-bundles, by the same proofs. As they have pointed out to me, any $R N_{2}$-bundle is concordant to a cone bundle by the Alexander trick, so the corresponding homotopy functors are the same.

The thickenings $W_{0}$ and $W_{1}$ of the $n$-manifold $M$ are concordant if there is a thickening $Q$ of $M \times I$ such that $W_{i}$ is a regular neighborhood of $M \times\{i\}$ rel $\partial M \times\{i\}$ in $\partial Q, i=0,1$ (cf. [4]).

THEOREM 3. If $M$ is a compact $n$-manifold, $\xi \mapsto E(\xi)$ induces a bijection between $\mathcal{H}(M)$ and concordance classes of codimension 2 thickenings of $M$.

Proof. Every thickening of $M$ is in fact equivalent to $E(\xi)$ for some $\xi$ over $M$, by Lemma 3. On the other hand, given $\xi_{0}$ and $\xi_{1}$, and a concordance $Q$ between $E\left(\xi_{0}\right)$ and $E\left(\xi_{1}\right)$, a concordance between $\xi_{0}$ and $\xi_{1}$ can be constructed as a regular neighborhood of $M \times I$ in $Q$, by the relative version of Lemma 1.

It follows that the classifying space $\mathcal{G}$ is (canonically homotopy equivalent with) Cappell and Shaneson's classifying space $B R N_{2}$ [3], [4]. In the same way, "oriented" 2-cone bundles are classified by $B S R N_{2}$, and 2-cone bundles which are blockbundles on the $(k-1)$-skeleton are classified by $B R N_{2, k}$.

ReMARKs. (1) The Noguchi obstruction $\mathfrak{n}$ can be viewed as a natural transformation from $\mathcal{H}(\cdot)$ to $H^{2}(\cdot ; \gamma)$, since $\mathfrak{n}(\xi)$ depends only on the concordance class of $\xi$. Furthermore, $\mathfrak{n}(\xi)=0$ if and only if $\xi$ is concordant to a cone bundle which is a blockbundle on the 2-skeleton (cf. [4, §3]).

(2) In [4], Cappell and Shaneson completely determine the homotopy type of $B S R N_{2}$. An interesting problem is to give a geometric description of the resulting $H$-space structure on $B S R N_{2}$. 
3. Collared complexes. A collared complex $C$ on a polyhedron $X=\mid \mathcal{\text { is a }}$ locally finite covering of $X$ by compact subpolyhedra, together with a subpolyhedron $\delta \alpha$ of each element $\alpha$ of $C$ such that

(i) for each $\alpha \in \mathcal{C}, \delta \alpha$ is a union of elements of $C$,

(ii) if $\alpha$ and $\beta$ are distinct elements of $\mathcal{C}, \alpha^{\circ} \cap \beta^{\circ}$ is empty, where $\alpha^{\circ}=\alpha \backslash \delta \alpha$,

(iii) $\delta \alpha$ is collared in $\alpha$ for each $\alpha \in \mathcal{C}$. ((i) and (ii) imply that $\alpha \cap \beta$ is a union of elements of $\mathcal{C}$.)

Collared complexes are Akin's "general complexes" [1]. Examples of collared complexes are cell complexes, manifold complexes [5], and cone complexes [7].

The usefulness of collared complexes comes from the following proposition, derived from the proof of a lemma of Cohen and Sullivan [5, p. 142]. (See also [2, p. 21] and [7, p. 278].)

If $\mathcal{C}$ is a collared complex and $\alpha \in \mathcal{C}$, let $L(\alpha)$ be the geometric realization of the nerve of the finite partially ordered set $\{\beta \in \mathcal{C}, \alpha<\beta\}$, where $\alpha<\beta$ means $\alpha \subset \delta \beta$.

Proposition 1. If $\mathcal{C}$ is a collared complex on $X, \alpha \in \mathcal{C}$, and $x \in \alpha^{\circ}$, then

$$
l k(x ; X, \alpha) \cong(L(\alpha) * l k(x ; \alpha), l k(x ; \alpha)),
$$

where $l k$ denotes the link, and * denotes the join.

Proof. Use induction on the "depth" of $\alpha$, i.e. the length of a maximal chain $\alpha<\alpha_{1}<\cdots<\alpha_{n}$ in $\mathrm{C}$.

With this proposition, we can prove Lemma 2, by induction on the dimension of the base. Let $\xi / K$ be a cone bundle, with $|K|$ a manifold (with boundary). If $\sigma_{i} \in K$, and $\beta_{i}$ is the block of $\xi$ over $\sigma_{i}$, let $\delta \beta_{i}=E\left(\xi \mid \partial \sigma_{i}\right)$. By induction hypothesis, $\delta \beta_{i}$ is a codimension 0 submanifold of $\partial \beta_{i}$, so $\delta \beta_{i}$ is collared in $\beta_{i}$. Thus the set of blocks of $\xi$ forms a collared complex $\mathcal{C}$ on $E(\xi)$. The map $\beta_{i} \mapsto \sigma_{i}$ is an incidence preserving bijection between $\bigodot$ and $K$. Therefore $L\left(\beta_{i}\right)=L\left(\sigma_{i}\right)$ for all $\sigma_{i} \in K$. Thus the proposition implies $E(\xi)$ is a manifold (with boundary), since each block $\beta_{i}$ is a manifold and $|K|$ is a manifold.

4. Geometry of codimension 2 thickenings. Let $W$ be a codimension 2 thickening of the compact $n$-manifold $M$. If $x \in M$, the intrinsic dimension $d(x ; W, M)$ is the smallest integer $k$ such that $x$ is in the $k$-skeleton of every (PL) cell complex on $W$ which has $M$ as a subcomplex. The $k$ th intrinsic stratum $S_{k}$ of $M$ in $W$ is

$$
\{x \in M \backslash \partial M, d(x ; W, M)=k\} \cup\{x \in \partial M, d(x ; \partial W, \partial M)=k-1\} \text {. }
$$

(Cf. [12, p. 13]. Recall that $(\partial W, \partial M)$ is collared in $(W, M)$.) $S_{k}$ is a $k$-dimensional submanifold of $M$, and $\operatorname{cl}\left(S_{k}\right)=\cup_{j<k} S_{j} . S_{n}$ is the set of 
locally flat points of $M$ in $W$, and $S_{k}$ can be thought of as the points at which the "degree of local knottedness" of $M$ in $W$ is $n-k$. Let $\mathcal{S}=\left\{S_{k}\right\}$ denote this intrinsic stratification of $M$ in $W$.

By Lemma 3, we can assume that $W=E(\xi)$ for some 2-cone bundle $\xi / K$, $|K|=M$. Now for each block $\beta$ of $\xi$, choose a cellular subdivision of the "rim" $\beta^{\circ}=\operatorname{cl}(\partial \beta \backslash \delta \beta)$. These cells, together with the blocks themselves, form a cell complex $\mathcal{C}$ on $W$. Choose a cone structure for each cell of $e$ so that $\sigma_{i}$ is a subcone of $\beta_{i}$ for each cell $\sigma_{i} \in K$. (N.B. $K$ is not a subcomplex of $C_{\text {.) }}$ Then the dual cone complex $e^{*}$ on $W$ [7] will have the complex $K^{*}$ on $M$ as a subcomplex. (Note that the cones of $\bigodot^{*}$ are cells, but if $\alpha \in C^{*}$ and $\alpha \cap \partial W \neq \varnothing$, the apex of $\alpha$ lies in $\partial W$.) It follows that $\operatorname{cl}\left(S_{k}\right)$ is a subcomplex of $K^{*}$ for all $k$, i.e. the cells of $K$ are transverse to the intrinsic stratification of $M$ in $W$. (See [7, p. 287] for a discussion of transversality to a stratification.)

If $K^{\prime}$ is a subdivision of $K$, the cone bundle $\xi^{\prime} / K^{\prime}$ is a subdivision of $\xi / K$ if for each $\sigma_{i} \in K, \beta_{i}(\xi)=\cup \beta_{i}\left(\xi^{\prime}\right)$, where the union is taken over all blocks $\beta_{j}\left(\xi^{\prime}\right)$ over cells $\tau_{j} \in K^{\prime}$ such that $\tau_{j} \subset \sigma_{i}$.

It is not hard to see that a subdivision $\xi^{\prime}$ of $\xi$ will exist over the subdivision $K^{\prime}$ of $K$ if and only if $\left(K^{\prime}\right)^{*}$ extends to a cell complex on $W=E(\xi)$ (for some cone structuring of $K^{\prime}$ ). This is equivalent to the condition that $K^{\prime}$ be transverse to the intrinsic stratification $\mathcal{S}$. Therefore, $\xi$ can be restricted to precisely those subpolyhedra of $M$ which are transverse to $\delta$.

Thus the fact that concordance classes of cone bundles can be "pulled back" is a consequence of the geometric fact that any subpolyhedron $X$ of the manifold $M$ can be moved transverse to $\delta$. (In fact, Stone's transversality theorem [12] can be easily proved from the mockbundle viewpoint-cf. [7, p. 287].)

The following result is important in [4].

Proposition 2. Let $W$ be a codimension 2 thickening of $M$, and let $N$ be a locally flat codimension $q$ submanifold of $M$, with $\partial M \cap N=\partial N$. Suppose that $N$ is transverse to the intrinsic stratification $\delta$ of $M$ in $W$. Then there is a cone bundle $\xi$ over $M$ with $E(\xi)=W$, and a normal blockbundle $\nu$ of $N$ in $M$ such that $E(\nu)$ is transverse to $\mathcal{S}$, and $E(\xi \mid E(\nu))$ is a codimension $q$ thickening of $E(\xi \mid N)$ equivalent to $E\left(q^{*} v\right)$, where $q: E(\xi \mid N) \rightarrow N$ is a homotopy inverse of the inclusion.

Proof. $N$ is transverse to $\delta$ implies there is a cone bundle $\eta / L,|L|=M$, with $E(\eta)=W$ and $N$ a subcomplex of $L$. Let $K$ be the canonical "full" subdivision of $L$ constructed in [7, p. 276], and let $\xi$ be a subdivision of $\eta$ over $K$. (It is easy to construct $\xi$ explicitly.) Then the union of the cells in $K$ which meet $N$ is a regular neighborhood of $N$, and so this neighborhood equals $E(\nu)$ for some blockbundle $\nu$ over $N . E(\nu)$ is transverse to $\delta$ since it is a 
subcomplex of $K . E(\xi \mid E(\nu))$ is a manifold by Lemma 2, and it collapses to $E(\xi \mid N)$ since $E(\nu)$ collapses to $N$. Thus $E(\xi \mid E(\nu))$ is a thickening of $E(\xi \mid N)$. $E(\xi \mid N)$ is locally flat in $E(\xi \mid E(\nu))$ by Proposition 1, since the given collared complexes on $E(\xi \mid E(\nu))$ and $E(\nu)$ are abstractly isomorphic. Thus $E(\xi \mid E(\nu))$ $\supset E(\xi \mid N)$ is equivalent to $E\left(q^{*} \nu\right) \supset E(\xi \mid N)$ by the uniqueness of regular neighborhoods.

\section{REFERENCES}

1. E. Akin, Transverse cellular mappings of polyhedra, Trans. Amer. Math. Soc. 169 (1972), 401-438. MR 48 \#5088.

2. S. Buoncristiano, C. P. Rourke and B. J. Sanderson, A geometric approach to homology theory, London Math. Soc. Lecture Notes Series, no. 18, Cambridge Univ. Press, London, 1976.

3. S. E. Cappell and J. L. Shaneson, Nonlocally flat embeddings, smoothings, and group actions, Bull. Amer. Math. Soc. 79 (1973), 577-582. MR 47 \# 9635.

4. , Piecewise linear embeddings and their singularities, Ann. of Math. 103 (1976), $163-228$.

5. M. M. Cohen and D. Sullivan, On the regular neighborhood of a two-sided submanifold, Topology 9 (1970), 141-147. MR 41 \#262.

6. T. Matumoto and Y. Matsumoto, The unstable difference between homology cobordism and piecewise linear bundles (to appear).

7. C. McCrory, Cone complexes and PL transversality, Trans. Amer. Math. Soc. 207 (1975), 269-291.

8. C. P. Rourke, Block structures in geometric and algebraic topology, Actes Congres Internat. Math. (Nice, 1970), Vol. 2, Gauthier-Villars, Paris, 1971, pp. 127-132.

9. C. P. Rourke and B. J. Sanderson, Block bundles. I, Ann. of Math. (2) 87 (1968), 1-28. MR 37 \#2234a.

10. _ , $\Delta$-sets. I: Homotopy theory, Quart. J. Math. Oxford Ser. (2) 22 (1971), 321-338. MR 45 \#9327.

11. __ Introduction to piecewise-linear topology, Ergebnisse Math., Band 69, SpringerVerlag, Berlin and New York, 1972. MR 50 \#3236.

12. D. Stone, Stratified polyhedra, Lecture Notes in Math., vol. 252, Springer-Verlag, Berlin and New York, 1972. MR 51 \# 9074.

Department of Mathematics, Brown University, Providence, Rhode Island 02912 